\title{
Latest improvements in field deployable compound specific isotope analyzer based on quantum cascade lasers and hollow waveguide
}

Sheng Wu, Andrei Deev

Sheng Wu, Andrei Deev, "Latest improvements in field deployable compound specific isotope analyzer based on quantum cascade lasers and hollow waveguide," Proc. SPIE 8993, Quantum Sensing and Nanophotonic Devices XI, 89931W (31 January 2014); doi: 10.1117/12.2041110

SPIE. Event: SPIE OPTO, 2014, San Francisco, California, United States 


\title{
Latest improvements in field deployable compound specific isotope analyzer based on quantum cascade lasers and hollow waveguide
}

\author{
Sheng Wu, Andrei Deev \\ PEER Institute \\ 738 Arrow Grand Circle, Covina, CA 91722
}

\begin{abstract}
In our development of a field deployable infrared isotope ratio spectrometer (IR2) for compound specific isotope analysis (CSIA), the IR2's accuracy and stability have reached a stage that small fractionation in reference gas pulses generated as standard are observed easily and repeatedly. Such fine fractionation is often difficult to observe in regular Isotope Ratio Mass Spectrometer (IRMS) due to instrument drift on the order of $10 \mathrm{~s}$ of minutes. After careful design of reference pulse sequences and long period of data collection, such fractionations are also verified in a commercial continuous flow CSIA-IRMS. Implications of the fractionation and observation process are discussed.
\end{abstract}

\section{KEY WORD LIST}

green photonics, quantum cascade laser; laser spectroscopy, Hollow Waveguide; Compound Specific Isotope Analyzer

\section{INTRODUCTION}

Field deployable compound specific isotope analysis (FD-CSIA) has many applications in fossil energy exploration, food and drug identification, environmental protection and human health care. We have been developing such an instrument based on hollow waveguide (HWG) and mid-Infrared quantum cascade (QC) lasers, i.e. Infrared Isotope Ratio (IR2) spectrometer which is based on absorption spectroscopy and coupled with gas chromatograph (GC) to realize FD-CSIA instrument[1, 2]. Instruments for individual gas isotope analysis, e.g. $\mathrm{CO}_{2}$ and $\mathrm{H}_{2} \mathrm{O}$, based on optical absorption spectroscopy have already been commercialized, and they have demonstrated better field operational capabilities. One of the advantages of such optical absorption based instrument is to be able to conduct measurement without frequent reference calibration[3], and this advantage lies in the fact that the Beer's law that this kind of instruments rely on which could effectively calibrate out drifts which otherwise will be hard to remove with mass spectrometry based instruments. For practical application, it is also desirable for conventional optical absorption based instrument to operate with minimal calibration because the sample cells usually are large, i.e. 10s or even 100s of $\mathrm{ml}$ and frequent calibration will take much longer time and consume lots of reference gases. The large sample cell volume also means such optical absorption based platform is not compatible with capillary based chromatographs, where the sample volume is often limited to $\mu \mathrm{L}$ or less. In principle, our IR2 spectrometer and resulting CSIA instrument should also require less frequent calibration, although the small sample cell volume, i.e. 100s of $\mu 1$, enables fast calibration with minimal consumption of reference gases. Such small sample cell volume, a unique feature of HWG and QC laser based platform, could make Mid-Infrared optical absorption based spectrometer compatible with capillary chromatographs. This unique feature could improve the detection limit of GC-IR (Infrared) close to that of GC-MS (mass spectrometery), and at the same time requiring minimal internal standard or calibration.

In stable isotope analysis, pulses of reference gases are used for calibration all the time to ensure the integrity, stability of the measurement over long period of time. This is especially vital for Isotope Ratio Mass Spectrometer (IRMS), and in fact, the classic dual inlet offline IRMS has an accuracy almost an order of magnitude better than GC coupled online IRMS because the dual inlet IRMS does more frequency calibration than online IRMS which only calibrate at the end or the start of a $\mathrm{GC}$ run, i.e. every 10 minutes to an hour.

Quantum Sensing and Nanophotonic Devices XI, edited by Manijeh Razeghi, Eric Tournié, Gail J. Brown, Proc. of SPIE Vol. 8993, 89931W · (C) 2014 SPIE · CCC code: 0277-786X/14/\$18 - doi: 10.1117/12.2041110 
Here, our IR2 has demonstrated the stability and accuracy that enable easy observation of some fine fractionations during reference gas pulses generation; whereas such fractionations are hard to observe with online IRMS due to the relatively large drift associated with mass spectrometry.

\section{EXPERIMENT}

\section{Latest progresses in IR2 spectrometer reduce system weight and power consumption}

Recently, noticeable progress has been made in QC lasers in the range of $2,300 \mathrm{~cm}^{-1}$. The devices could now working in continuous wave $(\mathrm{CW})$ mode at above room temperature up to $70^{\circ} \mathrm{C}[4]$ and with power consumption less than $1 \mathrm{~W}[5]$. These results enable us to replace old $\mathrm{CW}$ QC lasers which need to be cooled down to $-20^{\circ} \mathrm{C}$, and the savings in Peltier cooling power translate into total saving in system temperature control power from $300 \mathrm{~W}$ to only $15 \mathrm{~W}$ ! In fact, we are no longer cooling the spectrometer box and QC lasers, and only small heating power is needed to maintain the spectrometer temperature. With better insulation, we expect the total power to maintain temperature of the spectrometer, including powering and temperature control for the laser, will be reduced to less than $12 \mathrm{~W}$ !.

With the HWG platform, we could pump down the IR2 spectrometer system with a pair of micro diaphragm pump, e.g. Hargraves CTS pump, while maintaining a sample refresh rate higher than $10 \mathrm{~Hz}$. These micro diaphragm pumps consume less than $5 \mathrm{~W}$ average power and weigh less than $200 \mathrm{grams}$.

Now, an IR2 spectrometer with total power consumption less than $20 \mathrm{~W}$ and weighs less than 10 pounds is being designed. This opens the possibility to transport the IR system to many places previously impossible, e.g. airborne systems on UAVs.

\section{Improved stability allows accurate measurement during a long GC run}

In the past 3 years, we have developed the online continuous flow $\mathrm{CO}_{2}$ Infrared Isotope Ratio (IR2) sensor module for GC using QC laser and HWG; we have succeeded in using a $\mathrm{CW}$ QC laser for simultaneously measuring ${ }^{12} \mathrm{CO} 2$ and ${ }^{13} \mathrm{CO} 2$ at $\sim 2,299.70 \mathrm{~cm}^{-1}$. The instrument is now able to measure $\delta^{13} \mathrm{C}$ with an error within \pm 0.2 per mil for a hydrocarbon compound coming out of a GC. One of the major challenges in coupling IR2 with GC is to ensure the stability of the IR2 spectrometer during a GC run lasting 10s of minutes, since reference gas calibration could not be practically carried out throughout the GC run, and only limited to the beginning or the end of the GC run. Here, we demonstrate the stability of the IR2 spectrometer is already comparable or at certain scenario better than IRMS, i.e. fine fractionation could be easily observed during reference gas pulse generation.

\section{RESULTS}

\section{IR2 spectrometer easily observed fractionation in reference pulse generation}

We programmed our GC-IR2 system with a GC run that has two reference pulses inserted before the samples coming out of the GC (see figure 1a). Accidental typo made the two reference pulses of different pulse widths, i.e. 60 seconds and 10seconds, and to our surprise, the values of the two pulses repeatedly differ by $\sim 0.25 \%$ o for several GC runs. The reference pulses are generated by an automated 3-way Swagelok ball valve with 1/8" tubing, which switch between GC elute mode and reference gas mode, and the reference pulse width is controlled by the GC-IR2 computer. The reference gases are $3 \%$ or $1 \% \mathrm{CO}_{2}$ buffered with pure air, and we could adjust the flow rate read on a Thorpe tube flow meter by changing the backing pressure and a needle valve.

We then proceed to verify this is indeed a real fractionation by testing GC run with reference pulses of different pulse widths only (figure 1b). In this GC run lasting 2,700 seconds, we group $4 \sim 8$ reference pulses of same pulse width together, i.e. 7 pulse width values of $10 \mathrm{sec}, 5 \mathrm{sec}, 20 \mathrm{sec}, 40 \mathrm{sec}, 80 \mathrm{sec}, 160 \mathrm{sec}$ and back to $10 \mathrm{sec}$ at the end of the GC run. The measured ${ }^{13 / 12} \mathrm{C}$ values are grouped according reference pulse widths, and fractionation according to pulse width is obvious (figure 2a). At low pressure and slow flow, the fractionation could be as large as 1\%o when the pulse width varies from 5 seconds to $>100$ seconds. 


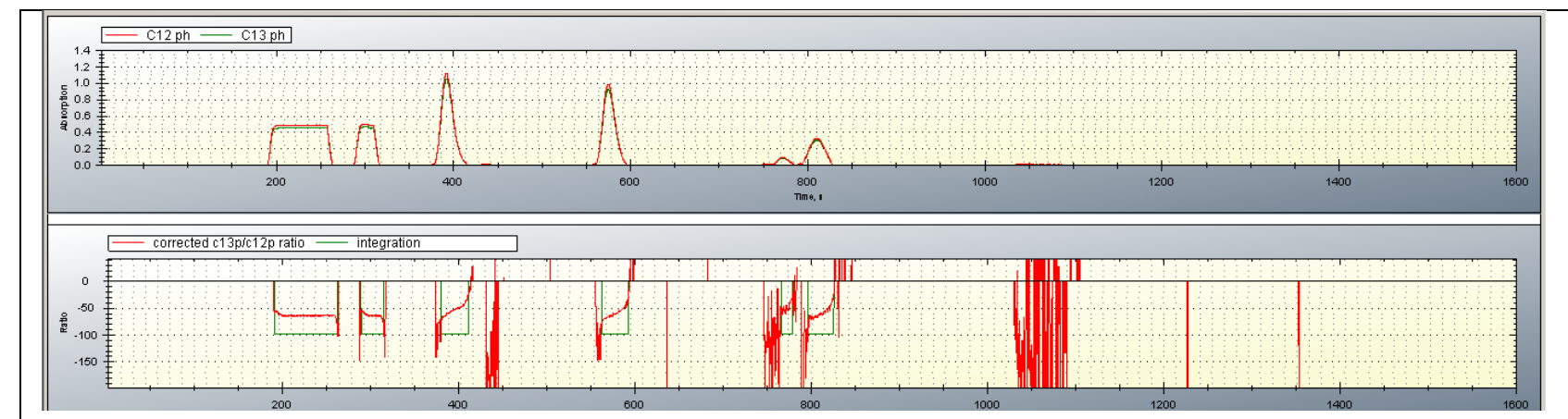

Figure 1a. GC run with two reference pulses ahead of the chemical elute peaks (starting at 400sec) for GC-IR2

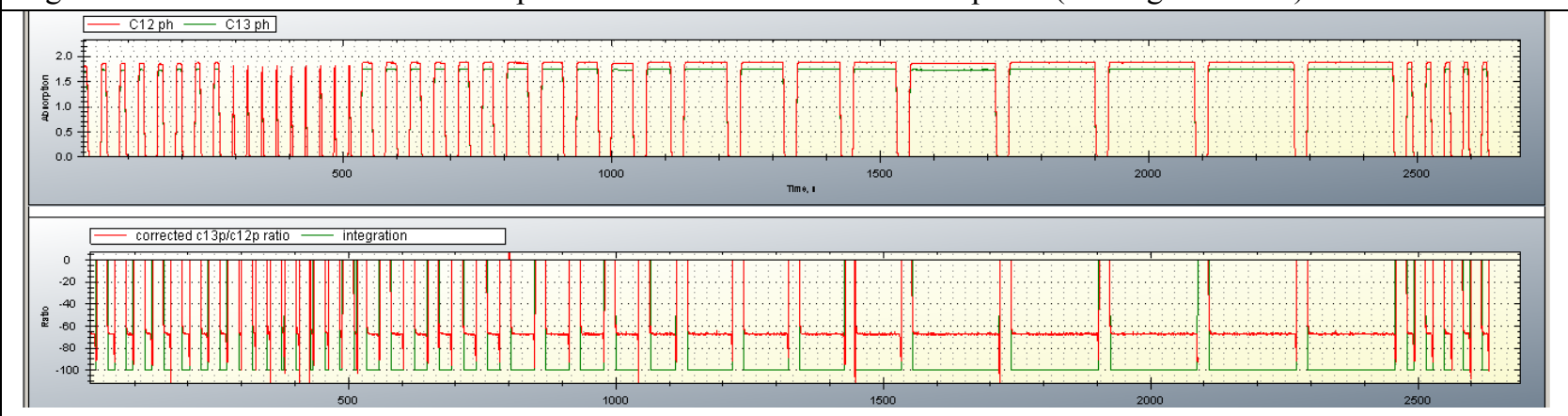

Figure 1b. GC run with reference pulses for IR2 which demonstrates pulse fractionation in Figure 2.

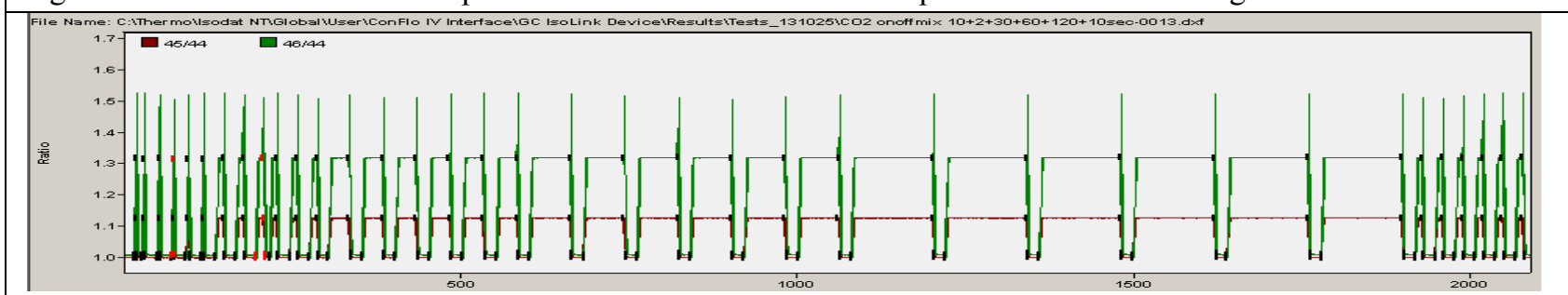

Figure 1c. GC run with reference pulses for Delta-V IRMS which could barely demonstrate pulse fractionation.

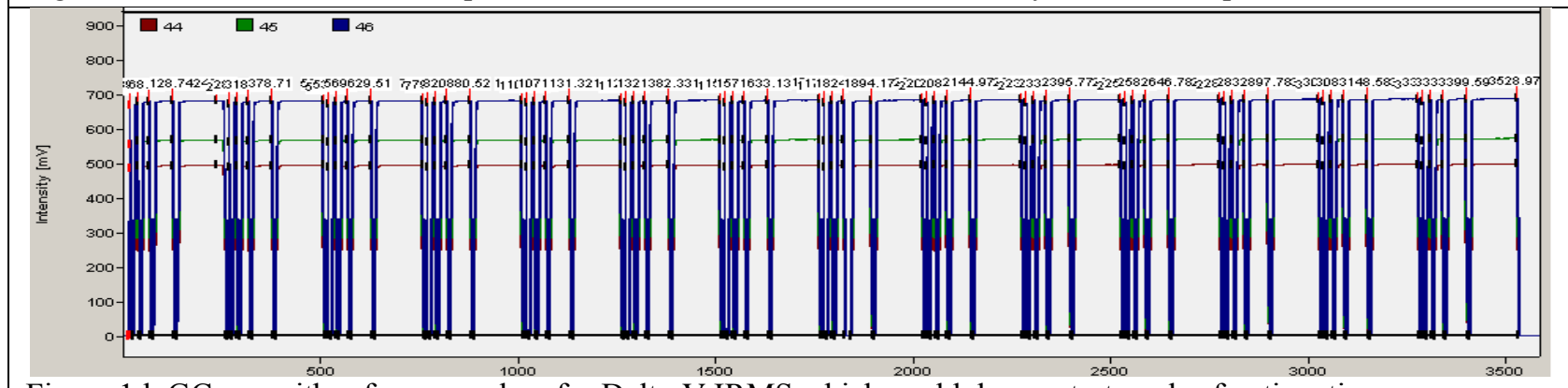

Figure 1d. GC run with reference pulses for Delta-V IRMS which could demonstrate pulse fractionation. 


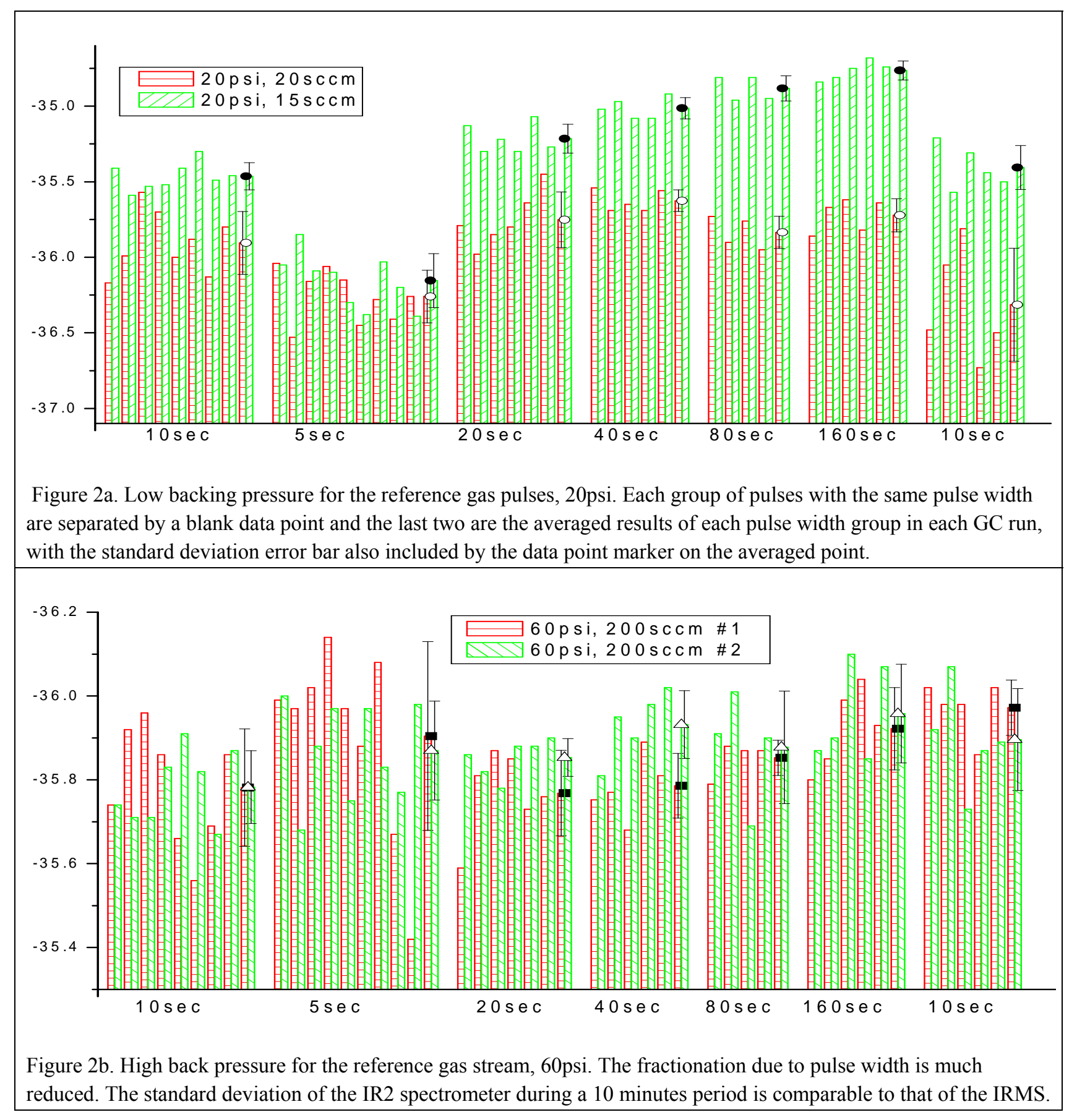

The pattern of fractionation also depends on the backing pressure and flow rate of the switching valve which generates the reference pulses. In general, the higher the backing pressure and faster flow will reduce the fractionation. The results are presented in figure $2 \mathrm{a}$ and $2 \mathrm{~b}$. The minimal observable fractionation is close to the standard deviation of the IR2 spectrometer, i.e. $0.3 \%$ o. This shows the accuracy and precision of the IR2 spectrometer are very close, i.e. the drift over an hour is close to its standard deviation in $<10$ minutes.

Then, we want to verify the existence of such fractionations versus reference pulse widths and generation conditions, i.e. pressure and dilution flow, in the standard GC-IRMS system (Thermo, Delta V). We first generated a series of 
reference pulses in a GC run for GC-IRMS system as shown in figure 1c. But we do not observe the same pattern in the measured results (figure 3a) as in our GC-IR2 system (figure 2a, and figure 2b).

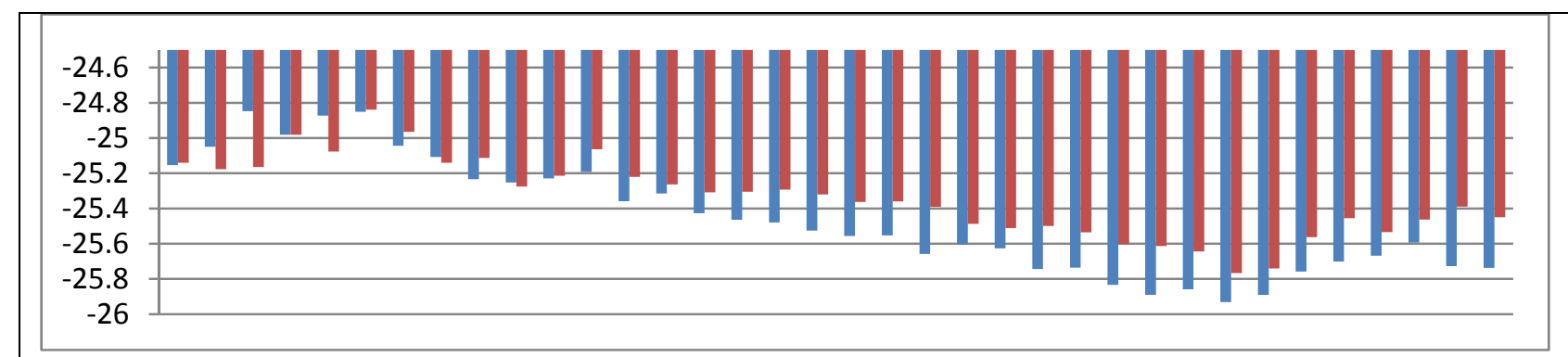

Figure 3a. Fractionation versus pulse width is not obvious with a series of reference pulses from Figure 1c. To GC runs with reference pulses in figure $1 \mathrm{c}$ are presented here.

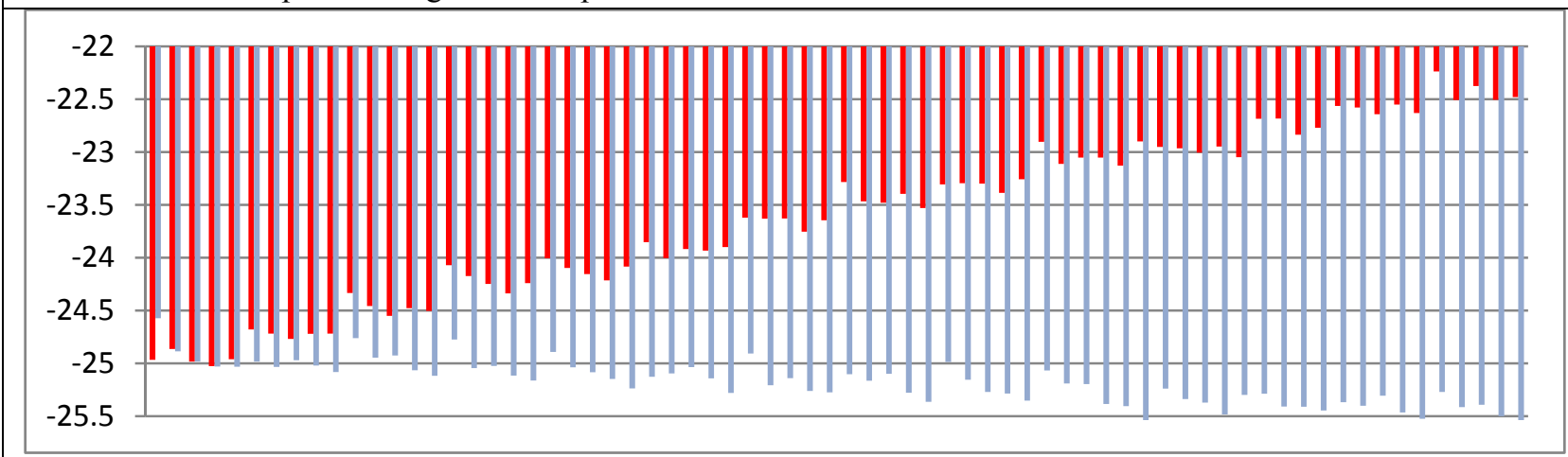

Figure $3 \mathrm{~b}$. Fractionation pattern is visible with reference pulses from Figure 1d.

The most obvious difference is that the last pulse group with the same shorter pulse width, i.e. 10sec, as the first group failed to reach the same ${ }^{13 / 12} \mathrm{C}$ values as the first group. This is related to the drift of the IRMS system in over 15 minutes, which is well known. In fact, this GC-IRMS mode, or online continuous flow (CF) measurement has a specified accuracy about an order of magnitude worse than constant calibration mode, i.e. dual beam mode IRMS[6]. Schemes to moderate such drift, e.g. bring in a reference on a more regular basis, i.e. every 20 minutes[7], could improve the accuracy. Due to the drift over an hour, the measurement results in figure 3 a do not unequivocally validate the fractionation during reference pulse generation as the pulse width changes. The drift is estimated to vary as large as $>1 \% \mathrm{o}$ in one hour period as we demonstrated below, therefore preventing the easy observation of the fractionations less than $0.5 \%$ o. To further verify such fractionations in GC-IRMS system, we applied a different kind of GC run with 5 reference pulses of 5 different pulse widths, i.e. $5 \mathrm{sec}, 10 \mathrm{sec}, 20 \mathrm{sec}, 50 \mathrm{sec}, 120 \mathrm{sec}$, in a consecutive group, and then repeat this group 12 times in about 1 hour time (figure 1d). The results of two such GC runs taken at 5 hours apart are shown in Figure 3b. We could see that there is an approximate repeating pattern of the ratio values of each group of 5 pulses; but there is a strong drift in baseline in one GC run while not that strong in the other GC run. To better illustrate the repeating pattern of the ratio values of each group of 5 pulses, we then average the results of these 12 groups in Excel sheet by summing up the reference pulses in each group with the same pulse width and divided by 12 . The results are shown in figure $4 \mathrm{a}$ through $4 \mathrm{~h}$ for different reference pulse generation conditions. The repeating patterns are now obvious, but come with a baseline shift. Each of the plots in figure 4 has two series, taken at different time usually separated over 6 hours in a day, which have baseline levels separated by as large as 1\%o, again a result of the drift of the IRMS system.

. To better illustrate the baseline drift of the GC-IRMS system, we also repeat this 1 hour GC run for over dozen times, i.e. over half a day. We characterized the system drift in a 24 hour period by plotting out the ratio of $\mathrm{m} / \mathrm{z} 45$ over $\mathrm{m} / \mathrm{z} 44$, as shown in figure $5 \mathrm{~b}$, which is the actual ratio of ${ }^{13 / 12} \mathrm{C}$ without calibration of the reference pulses at the 
beginning of each GC run. The drift plot in figure $5 \mathrm{~b}$ shows over $15 \%$ o change in 24 hour period, probably related to the $\sim 6^{\circ} \mathrm{C}$ temperature change of the room where the GC-IRMS is hosted. As a comparison, the GC-IR2 system, which is also hosted in a room with daily temperature variation $\sim 6^{\circ} \mathrm{C}$, the drift in actual ratio of ${ }^{13 / 12} \mathrm{C}$ is only $<1 \% \mathrm{o}$ as shown in figure 5a. There are several bumps with variation larger than $1 \%$ in figure $5 \mathrm{a}$ which lasts over 30 minutes, and they come in synch with the intentional sudden heating and cooling of the room temperature by over $5^{\circ} \mathrm{C}$ each way. We could see that the stability of the IR2 system during fast temperature change is obviously more robust than IRMS, and we expect the drift could be further reduced to less than 0.5 per mil even when we have $15^{\circ} \mathrm{C}$ swings each way, i.e. from $5^{\circ} \mathrm{C}$ to $35^{\circ} \mathrm{C}$, in room temperature.

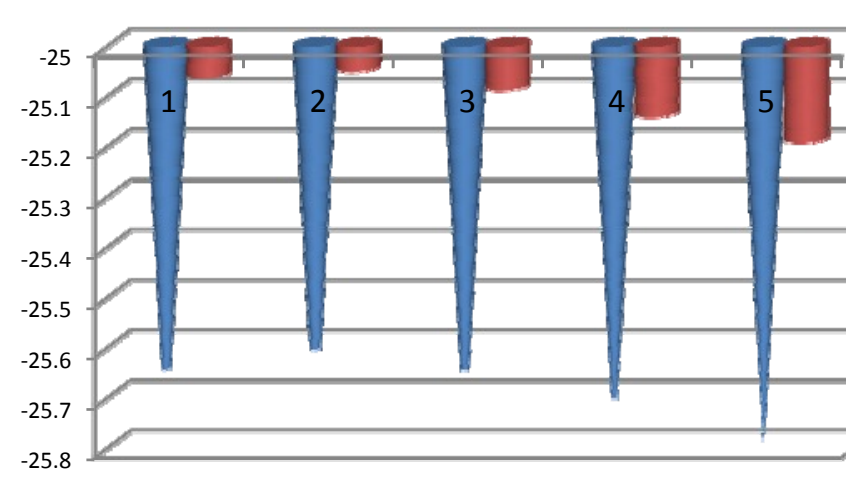

Fig. 4a. Reference pulse generated at $15 \mathrm{psi}, 5,000 \mathrm{mV}$ dilution

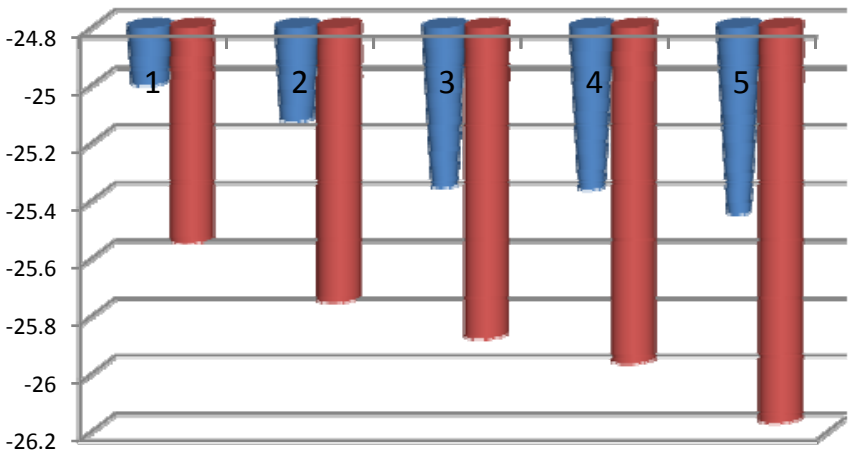

Fig. 4b. Reference pulse generated at $15 \mathrm{psi}, 500 \mathrm{mV}$ dilution

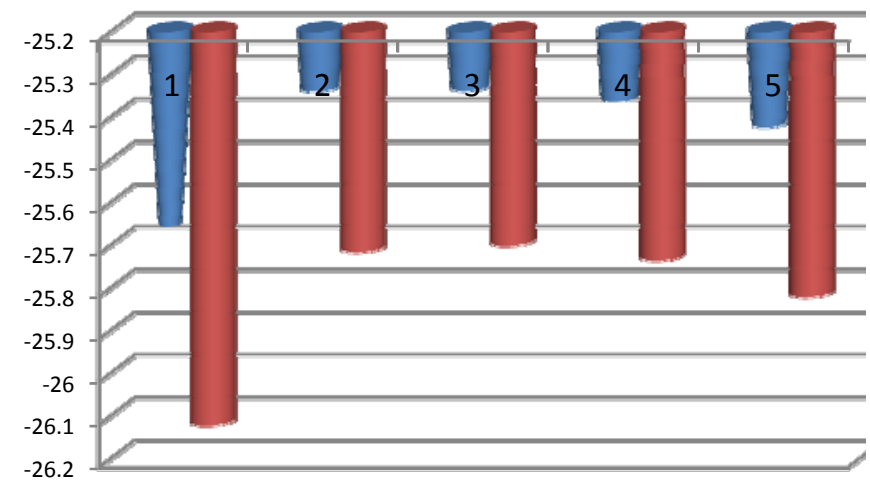

Fig. 4c. Reference pulse generated at 25psi, 5,000mV dilution

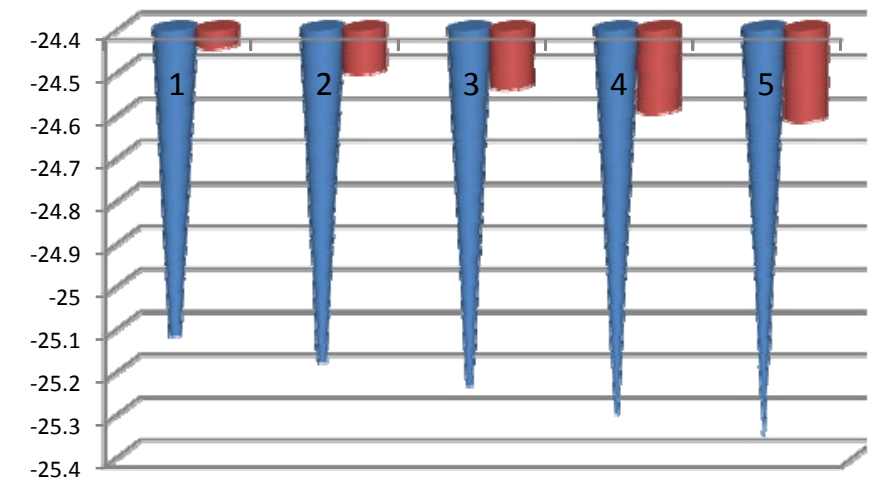

Fig. 4d. Reference pulse generated at 25psi, 500mV dilution

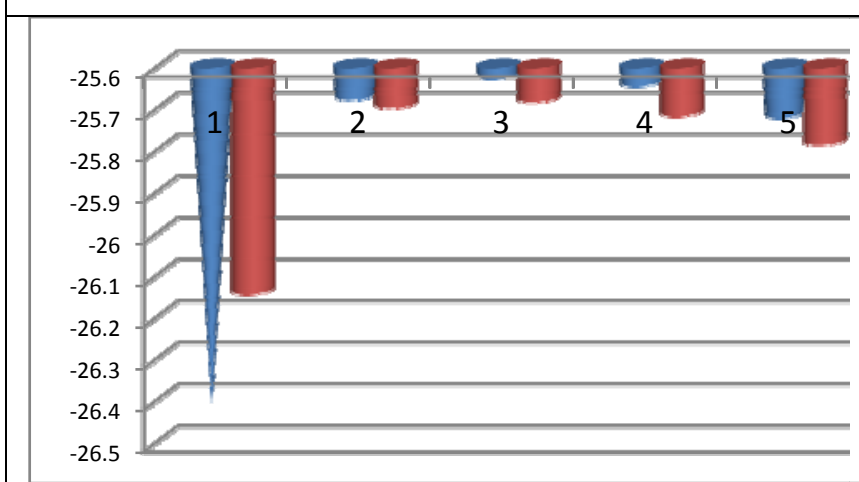

Fig. 4e. Reference pulse generated at 40psi, 5,000mV dilution

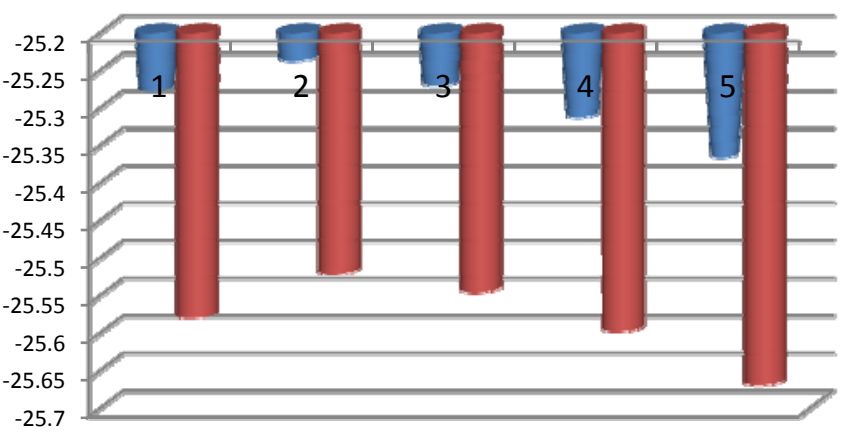

Fig. 4f. Reference pulse generated at $40 \mathrm{psi}, 500 \mathrm{mV}$ dilution 


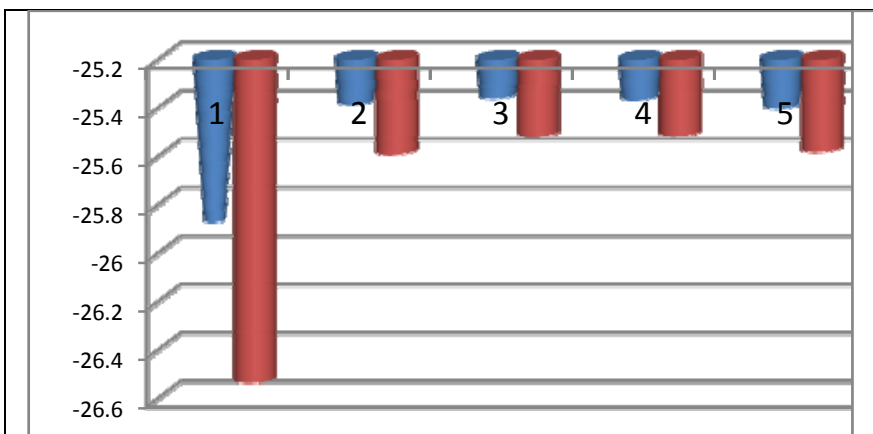

Fig. 4g. Reference pulse generated at 58psi, 5,000mV dilution.

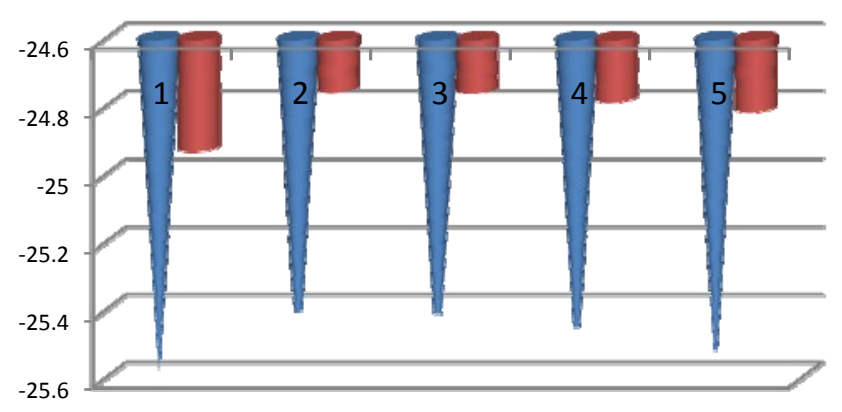

Fig. $4 \mathrm{~h}$. Reference pulse generated at $58 \mathrm{psi}, 500 \mathrm{mV}$ dilution

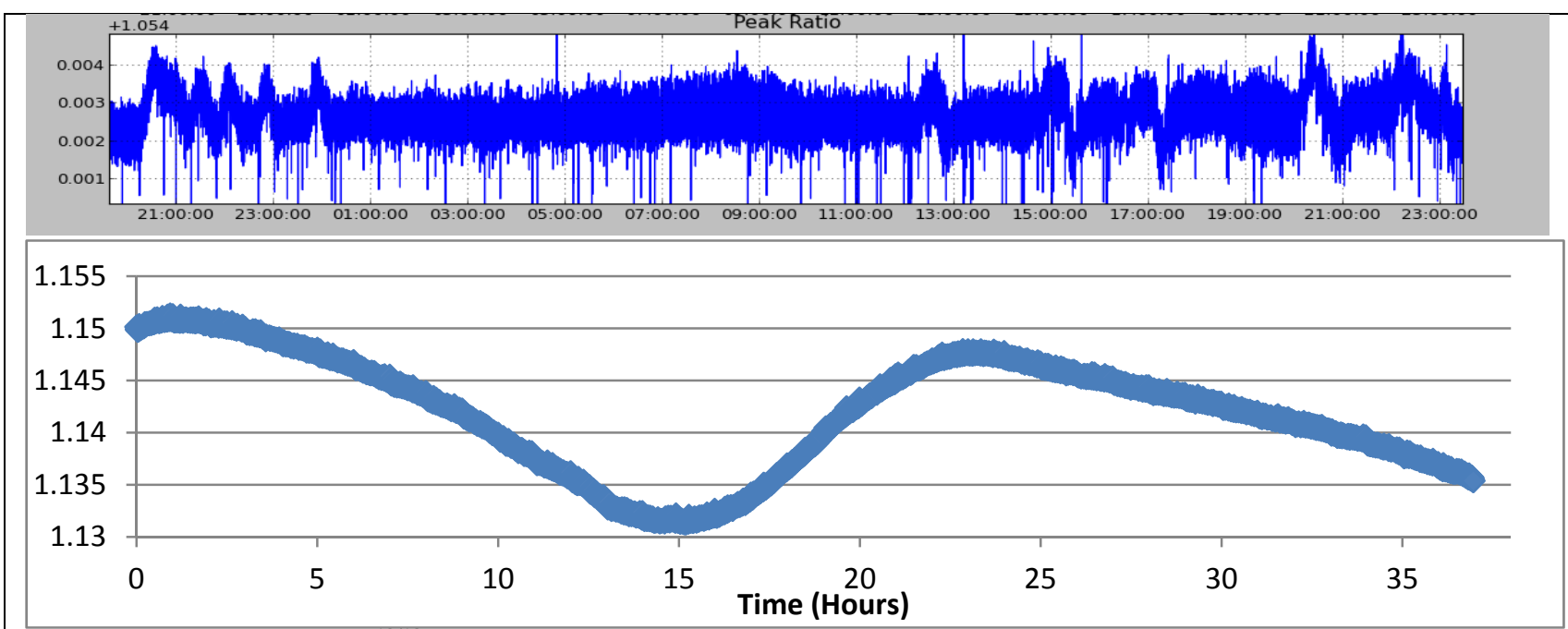

Top, figure 5a. Instrument ${ }^{13 / 12} \mathrm{C}$ drift before calibration measured on IR2 spectrometer over $\sim 28$ hours; Bottom, figure 5b. Instrument ${ }^{13 / 12} \mathrm{C}$ drift before calibration measured on IRMS over $\sim 36$ hours.

\section{DISCUSSIONS}

\section{Advantages and disadvantages of the IR2 sensor}

IR2 spectrometer is based on strong optical absorption in the Mid-Infrared, therefore, it has advantages and disadvantages over IRMS. The advantages include the easy differentiation of isotopomers with the same mass, e.g. ${ }^{13} \mathrm{CO}_{2}$ versus ${ }^{12} \mathrm{C}^{17} \mathrm{O}^{16} \mathrm{O}$. But, it could not detect molecules without strong IR active absorption features, e.g. $\mathrm{H}_{2}$, or $\mathrm{H}_{2} \mathrm{O}$. The future development of room temperature $\mathrm{THz}$ lasers and detectors in the $200 \mathrm{~cm}^{-1}$ range could enable the detection of $\mathrm{H}_{2} \mathrm{O}$ there with IR2, but not yet practical right now.

The stability of the IR2 spectrometer is inherent due to the underlining optical absorption nature of the instrument. This inherent property lends to the easy observation of fractionation in reference pulses as the conditions of pulse generation are varied.

The precision of the IR2 spectrometer is also getting close to that of IRMS. Although the sample amount needed is still larger than MS, it is already below $10 \mu \mathrm{L}$ (Standard Pressure and Temperature), making it compatible with GC operation. The small volume makes calibration easy to carry out in the field. The small sample volume, i.e. $<10 \mu \mathrm{L}$ (standard temperature and pressure, STP) makes our IR2 spectrometer unique when compared to other well-known 
optical absorption based instruments with larger sample volumes, e.g. cavity enhanced and multipass cell absorption spectroscopy platforms all have sample volume larger than $1 \mathrm{ml}$ STP.

\section{CONCLUSION}

We varied the reference pulse width, backing pressure and flow rate, and carefully measured the isotopic ratios of the reference gas pulses using both Isotope Ratio Mass Spectrometer (IRMS) and our newly developed Infrared Isotope Ratio (IR2) spectrometer. Results from both instruments demonstrated the existence of the variations in isotopic ratio as the pulse width, backing pressure and flow rate are varied. However, IR2 has better stability over longer period of time, i.e. $>1,000$ seconds, and therefore better divulge such variations, and give better guidance in generating more consistent and accurate reference pulses. The demonstrated stability translates directly into accuracy of the IR2 instrument, and bodes well for a calibration free or minimal calibration CSIA instrument which fits best for field deployment.

\section{ACKNOWLEDGEMENT}

We gratefully acknowledge the financial support from DOE-RPSEA project titled 'Novel Gas Isotope Interpretation Tools to Optimize Gas Shale Production' (DE-AC26- 07NT42677).

\section{REFERENCES}

1. Wu, S. and A. Deev, A Field Deployable Compound Specific Isotope Analyzer based on Quantum Cascade Laser and Hollow Waveguide. SPIE Photonics West Conference, Nano and Quantum Sensing, 2013, 2013.

2. Wu, S., et al., Hollow waveguide quantum cascade laser spectrometer as an online microliter sensor for gas chromatography. Journal of Chromatography A, 2008. 1188(2): p. 327-330.

3. Friedrichs, G., et al., Toward continuous monitoring of seawater 13CO2/12CO2 isotope ratio and pCO2: Performance of cavity ringdown spectroscopy and gas matrix effects. Limnol. Oceanogr. Methods 2010. 8: p. 539-551.

4. $\quad$ Feng, X., et al., Room Temperature CW Operation of Mid-IR Distributed Feedback Quantum Cascade Lasers for CO2,N2O, and NO Gas Sensing. Selected Topics in Quantum Electronics, IEEE Journal of, 2012. 18(5): p. 1605-1612.

5. Hinkov, B., et al., Singlemode quantum cascade lasers with power dissipation below $1 \mathrm{~W}$. Electronics Letters, 2012. 48(11): p. 646-647.

6. Brenna, J.T., et al., High-precision continuous-flow isotope ratio mass spectrometry. Mass Spectrometry Reviews, 1997. 16(5): p. 227-258.

7. Merritt, D.A., W.A. Brand, and J.M. Hayes, Isotope-ratio-monitoring gas chromatography-mass spectrometry: methods for isotopic calibration. Organic Geochemistry, 1994. 21(6 7): p. 573-583. 